\title{
Wireless monitoring of geotechnical and hydrology instrumentation using the battery-powered MineHop network
}

\author{
A Dulmage Mine Design Technologies Inc., Canada \\ M Trenwith Mine Design Technologies Inc., Canada
}

A Cervinka Newtrax Technologies Inc., Canada

\begin{abstract}
Technology advances in recent years have made it possible for battery-powered wireless communication to thrive in underground mining environments. SMART Geotechnical instruments from Mine Design Technologies, as well as vibrating wire based instruments from many manufacturers can now be monitored in near real time with the MineHop battery-powered network. This paper will discuss the different applications of the MineHop technology, the ease of installation and maintenance, hardware and software specifications, and examples of typical installations.
\end{abstract}

\section{$1 \quad$ Introduction}

The combination of heavy mobile equipment and the very tight nature of the operating space in an underground mine means that any hard wired communication system is inherently subject to damage by equipment, causing significant and ongoing maintenance issues. With advancements in both battery technology, as well as ultra-low power radio frequency (RF) microchips, the idea of a battery-powered wireless sensor network for use in extremely rugged environments such as underground mining, has been realised. The small nature of the batteries, enclosures and antennae, allows the equipment to be mounted in areas that pose very little risk of damage by heavy equipment. Since the batteries used are of a high capacity, and the power consumption of the equipment is so low, years of battery life can be achieved while maintaining real time monitoring of most types of geotechnical and hydrology instruments available today.

\section{$2 \quad$ System components and specifications}

\section{$2.1 \quad$ Overview}

The system is called the MineHop network by Newtrax Technologies Inc. It is comprised of a central server, often located on surface in a main office or network room, gateways (options for Ethernet or leaky feeder connectivity), and battery powered wireless nodes. The nodes can be either standard nodes used as repeaters, or remote telemetry units (RTUs) that are capable of reading different types of geotechnical and hydrology Instruments.

\subsection{Central server}

The central server acts as the central point for the MineHop Network by providing TCP/IP connectivity to all the MineHop gateways of the system. The web console allows users to view and manage the geotechnical and hydrology data coming from the MineHop wireless network and the associated sensors. It also provides real time information about the status of the MineHop infrastructure network, allowing for easier maintenance and repair. The server itself can be a physical rackmount computer (Figure 1), or a virtual 
machine that is imported to an existing virtual environment. The Central Server bridges the gap between MineHop RF network and the mine's Ethernet infrastructure.

The key features and benefits of the system include network inventory and visualisation, alarms and event collection, notifications, configuration, reports and dashboards, user security, and map management.

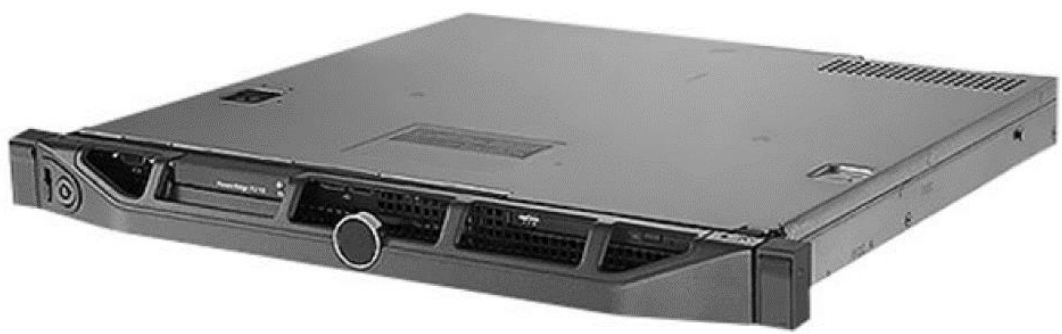

Figure 1 Newtrax central server (physical)

\subsection{Ethernet gateway}

The Ethernet gateway provides the communication pathway between the central server and the MineHop $\mathrm{RF}$ network. A gateway is commonly installed at each level of the mine. Its purpose is to relay the packets of data received from the MineHop RF network across the mine's Ethernet infrastructure back to the central server. It forwards the packets received on the RS-232 port from the wireless network to the Ethernet port that communicates with the central server. There are four ports on the Ethernet gateway (Figure 2): an Ethernet port, an RS-232 serial port, a power port (120 or 240VAC), and an RS-422 port.

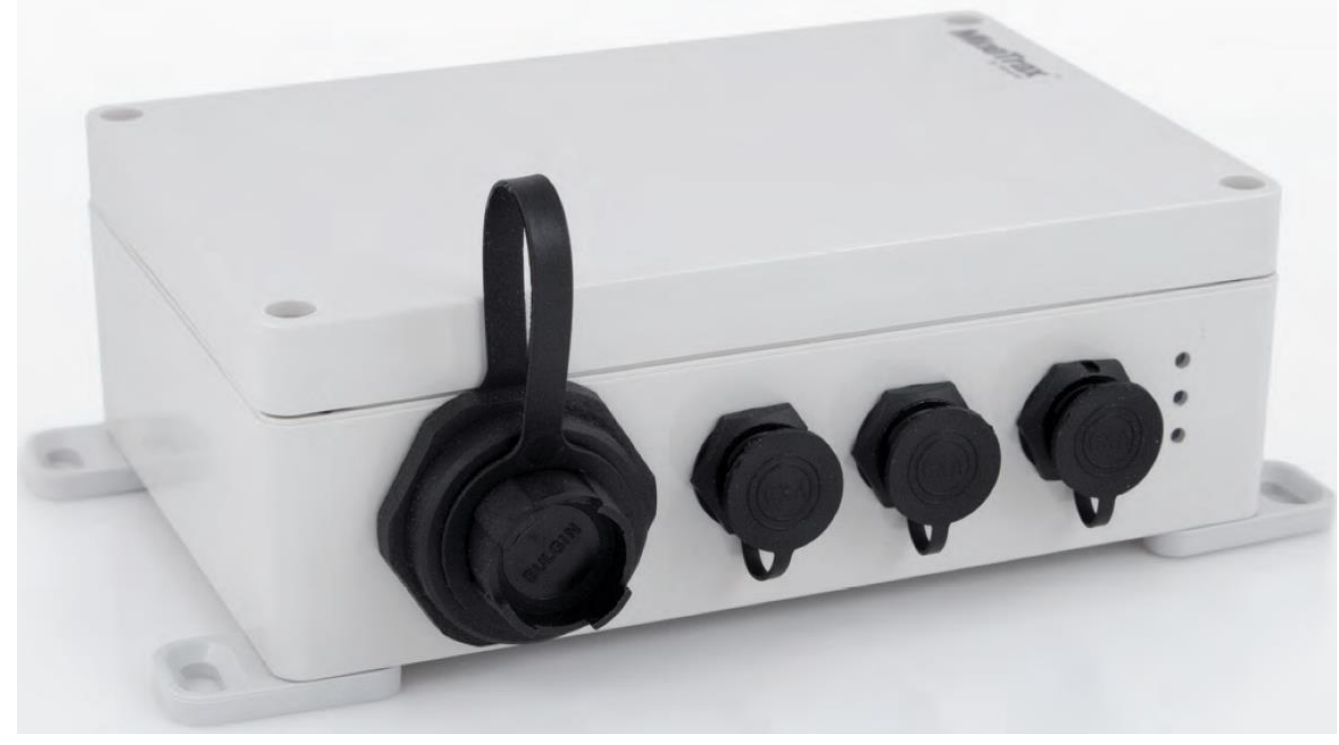

Figure 2 Standard Ethernet gateway

\subsection{Standard infrastructure node}

The standard infrastructure node provides mobile and backhaul routing capabilities for the system and can run on batteries for about three years. The RF chip is $902-928 \mathrm{MHz}$ and operates in the unlicensed $900 \mathrm{MHz}$ band, providing 50-150 m of typical range in an underground mining environment, or up to $600 \mathrm{~m}$ on surface. Each wireless node has a 32 bit unique identification number and operates on a pseudorandom hopping pattern utilising the time division multiple access (TDMA) and frequency-hopping spread spectrum (FHSS) protocols. A standard infrastructure node is shown in Figure 3. 


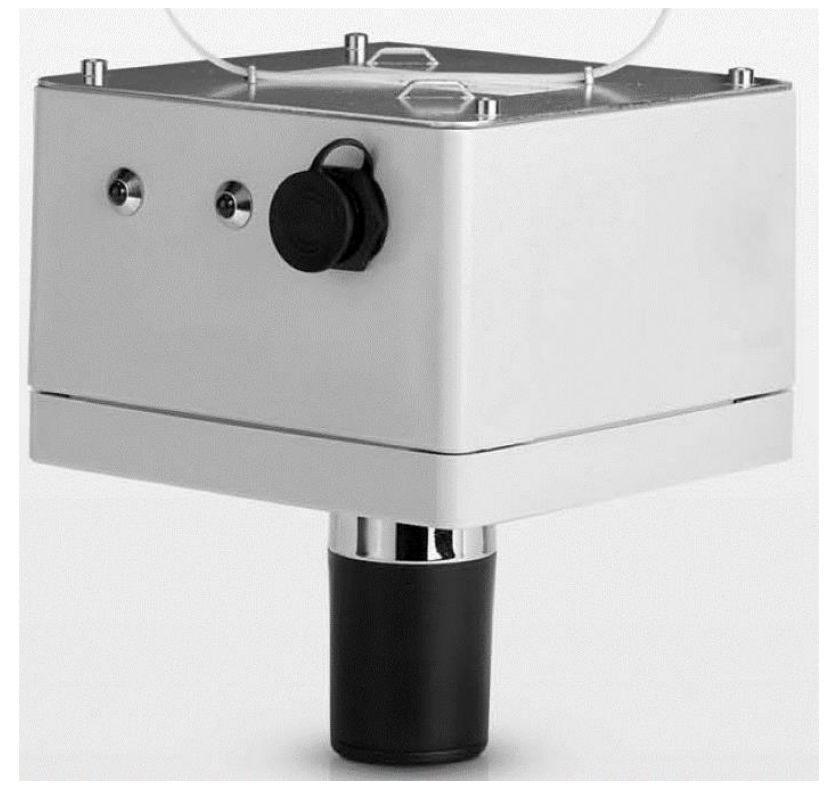

Figure 3 Standard infrastructure node

\subsection{MDT-RTU (Mine Design Technologies SMART)}

The MDT-RTU has the same RF properties as the standard node, but adds the unique functionality of reading Mine Design Technologies (MDT) patented SMART instruments. This includes Multi-Point Borehole Extensometers (MPBXs), SMART Cables, Contractometers, Ground Movement Monitors (GMMs) and Sloughmeters. Each MDT-RTU can read up to three MDT instruments as often as once per minute under normal configuration. The MDT-RTU (Figure 4) showing the RS-232 serial port and port one of three for an MDT SMART instrument. The remaining two ports are on the opposite side of the enclosure.

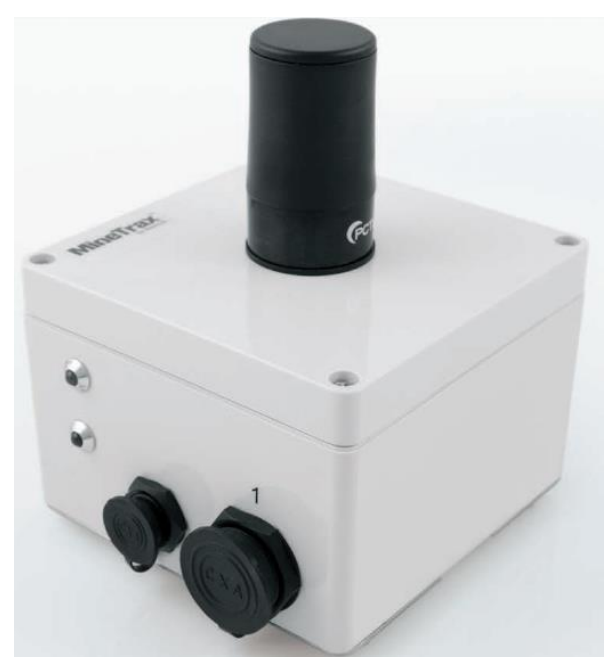

\section{Figure 4 MDT-RTU}

\section{$2.6 \quad$ VW-RTU (vibrating wire)}

The VW-RTU is similar to the standard node and the MDT-RTU, in that it is a battery-powered RF node, with the added capability of reading up to four vibrating-wire based instruments (with thermistors). There are many manufacturers of vibrating-wire instruments worldwide, several of which have been tested and proven compatible with the VW-RTU. There are two components to the VW-RTU: the main enclosure and the terminal box where the sensors are connected. These are shown in Figure 5. 


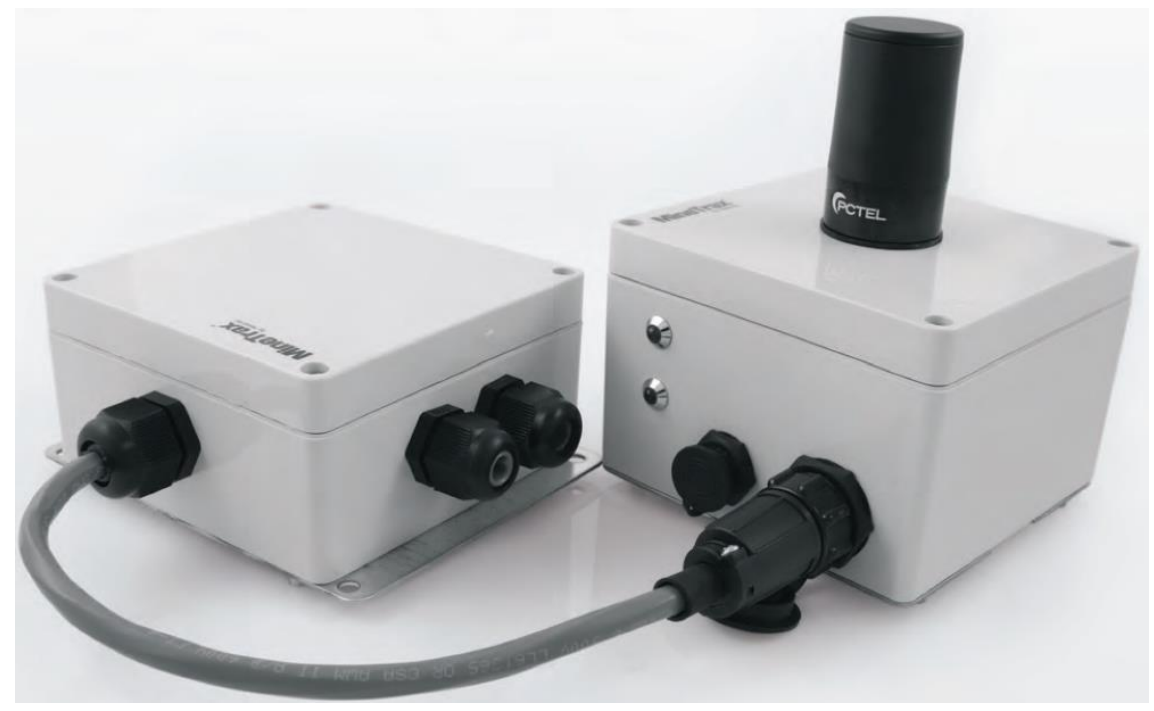

Figure 5 VW-RTU with terminal box

\subsection{SAA-RTU (Measurand Shape-Accel-Array)}

Newest to the MineHop network is the SAA-RTU, which can read up to four Measurand Shape-Accel-Array (SAA) instruments. It adds an external battery pack to power the Measurand Shape-Accel-Array and to maintain the typical three year maintenance free service period. Figure 6 shows the SAA-RTU and battery pack.

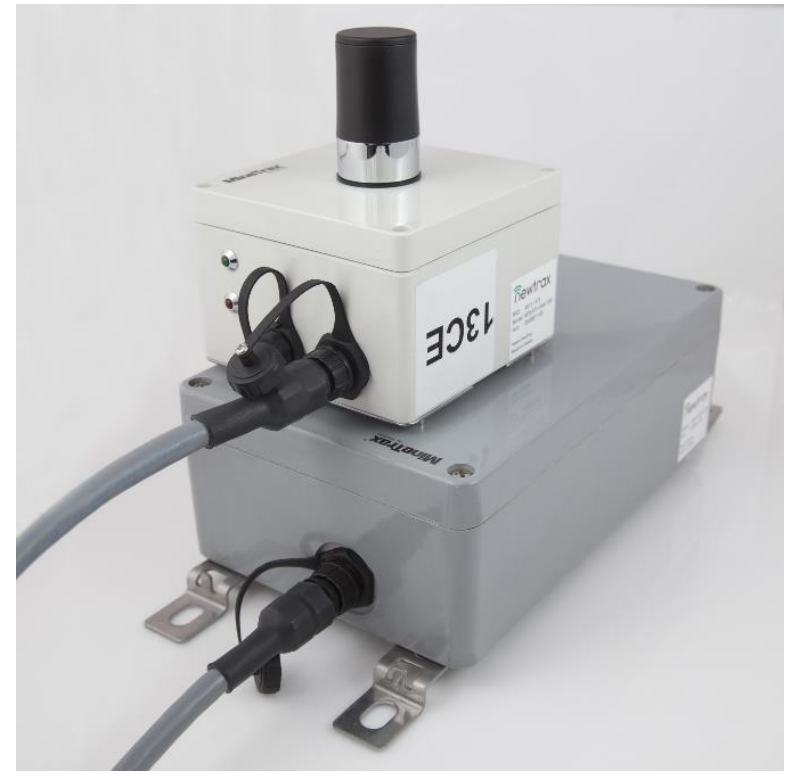

Figure 6 SAA-RTU with external battery pack

\section{$2.8 \quad \mathrm{RS} 485-\mathrm{RTU}$}

In addition to the new SAA-RTU is the RS485-RTU, which can communicate with RS-485 based devices. There are four dedicated RS-485 channels, each channel capable of supporting up to 32 individual devices. Depending on the power consumption of the RS-485 devices connected, several options are available to achieve several years of battery powered service. The RS485-RTU is shown in Figure 7. 


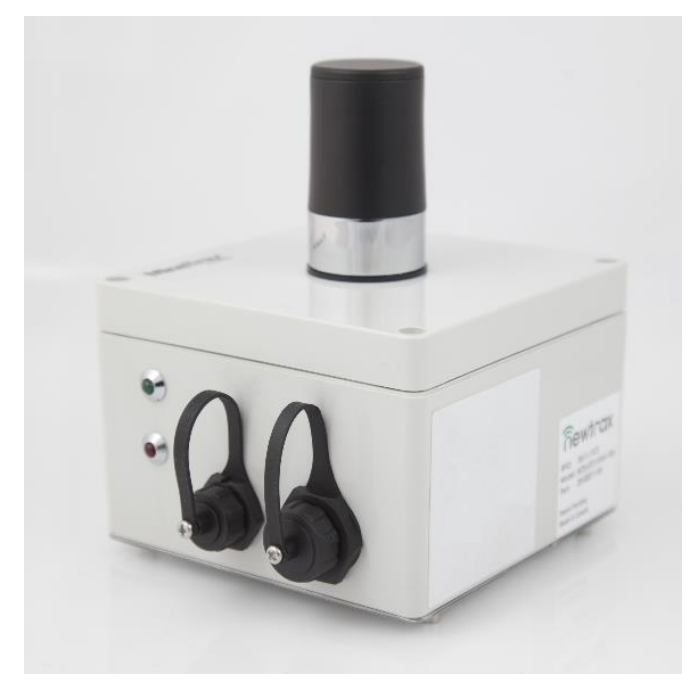

Figure 7 RS485-RTU

\section{Installation and data collection}

One of the main advantages of having a completely battery powered wireless backbone for geotechnical data is the ease of deployment. Since no wires are required for communication or power for the majority of the network, it typically takes hours to deploy rather than days, or even weeks.

The installation begins with the central server, which is often virtual, onto the company network. This is given a static IP address and configured with an SMTP server for sending email alarms and notifications. It provides a web interface that any authenticated user may login to for viewing data, acknowledging alarms, setting configuration parameters, as well as many other features.

The next phase is to install the first Ethernet gateway. This is often in an electrical substation underground on the level where the instrumentation is located. It also requires a static IP address and is configured to communicate with the central server. Since the Ethernet gateway can run on PoE power, only one CAT5 Ethernet cable is required for power and communication for this device. Once the Ethernet gateway has been powered up and there is communication with the central server, the wireless installation can begin.

The first wireless node is powered by removing a magnet on the bottom of the device. When the magnet is removed an internal switch is activated, further mitigating the risk of damage to the node by having an external switch. The node automatically scans the airspace for wireless 'neighbours' and connects to the neighbour, if one exists. The first RF node on the network is connected with a cable back to the Ethernet gateway and is typically called the 'sinkpoint', since all the data 'sinks' to this point.

The remaining nodes are powered up in the same fashion and automatically connect to the nearest neighbour. To assist with node placement $t$ user walks down the drift of the mine with a powered node and monitors the LED link lights on the enclosure to determine the range. The functionality of the link lights is described in Table 1. As the signal strength gets weaker, the node will tend to consume more power because of frequent disconnects and reconnects, and more scanning of the airspace for closer neighbours. In general, it is good practice to keep the LED lights in the green or blinking green range. The typical range between nodes in an underground environment is $50-150 \mathrm{~m}$. 
Table 1 LED light behaviour on wireless nodes and RTUs

\begin{tabular}{cc}
\hline LED light behaviour & Signal strength \\
\hline Green: SOLID, Red: SOLID & Node is scanning the airspace for neighbours \\
Green: SOLID, Red: OFF & Node has an excellent connection to a neighbour \\
Green: BLINKING, Red: OFF & Node has a good connection to a neighbour \\
Green: OFF, Red: BLINKING & Node has a poor connection to a neighbour \\
Green: OFF, Red: SOLID & Node does not have a connection to a neighbour
\end{tabular}

Once the area is reached where the geotechnical or hydrology instruments have been installed, the corresponding RTU is placed in the area and connected directly to the instruments so that data collection can begin. This RTU can be any combination of MDT, VW, SAA, or RS485 depending on the type of instruments that have been installed.

\section{$4 \quad$ Benefits of real time data}

Historically, mine personnel would visit each geotechnical or hydrology instrument on a regular basis to take a manual reading, perhaps once per week. Once an instrumentation program begins to grow, this can quickly become a full-time job for one or more employees. In some cases, instruments are too remote to read often, and the time between readings can slip to several months. This presents challenges for successful data interpretation, and inherently makes the data less useful.

Another option is to use data loggers that are semi or permanently fixed in an area and running cable back to the location, which is subject to damage by equipment. Employees would download the data from the loggers at specified intervals, but this would not provide real time data for quick decision-making.

By having data arriving every few minutes from all of the instruments in the mine, both surface and underground, engineers can take advantage of not only time savings compared to manual readings, but also more time to interpret and understand the data. Having early detection of potential structurally controlled falls of ground (FOG), enables a pre-emptive rehabilitation plan, which in turn assists the mine to execute the optimal mine plan or sequence. Ground failures may force the mine to step out of sequence, which can have a snowball effect on the stress conditions and therefore increase the risk of further FOG.

Furthermore, due to the simple nature of the system and deployment, there is little to no skilled labour required for system expansion. As new instruments are installed in the mine, they are easily connected and brought online without the need for running cables or scheduling other departments to install and maintain a wired infrastructure. Some installations have more than 200 wireless nodes between surface and several underground levels of the mine, and the longest run of nodes has more than 30 in a line. The only maintenance required on the system is a battery pack replacement, typically every three years. 